\title{
Effects of pelvic bone fracture on recurrence- free rate after bulbomembranous anastomotic urethroplasty in men with posterior urethral injuries
}

\author{
Don Kyoung $\mathrm{Choi}^{1}{ }^{\mathbb{D}}$, Sungjin $\mathrm{Kim}^{1}{ }^{10}$, Jong $\mathrm{Jin}_{\mathrm{Oh}^{2}}{ }^{\text {(iD }}$ \\ 'Department of Urology, Hallym University Kangnam Sacred Heart Hospital, Hallym University School of Medicine, Seoul, 'Department of Urology, Seoul National \\ University Bundang Hospital, Seoul National University College of Medicine, Seongnam, Korea
}

Purpose: To determine the impact of pelvic bone fracture on the recurrence of urethral stenosis after bulbomembranous anastomotic urethroplasty.

Materials and Methods: A total of 197 patients with complete posterior urethral injuries underwent bulbomembranous anastomotic urethroplasty. These patients were divided into two groups according to the presence of pelvic bone fracture. Recurrence of urethral stenosis was defined as the need for any postoperative surgical intervention. The surgical outcomes and postoperative recurrence rate of urethral stenosis were compared between the two groups, and significant predictors for posterior urethral restenosis, including pelvic bone fracture, were analyzed via multivariate analysis.

Results: Of the patients, 92 had pelvic bone fractures and the other 105 patients did not. The patients with pelvic bone fracture had increased involvement of the prostatic urethra compared to the group without pelvic bone fracture (3.8\% vs. $17.4 \%, p=0.002)$. Recurrence of urethral stenosis was more common in the pelvic bone fracture group (42/92, 45.7\%) than the group without pelvic fracture $(27 / 105,25.7 \%)$. In a Kaplan-Meier analysis, the recurrence rate at 5 years was significantly lower in the pelvic bone fracture group $(59.1 \%$ vs. $72.6 \%, p=0.003)$. A Cox proportional hazard analysis showed that the presence of pelvic bone injury was a significant predictor of posterior urethral re-stenosis.

Conclusions: Patients with posterior urethral injuries associated with pelvic bone fracture had a higher recurrence rate of urethral stenosis after bulbomembranous anastomotic urethroplasty than those without pelvic bone fracture.

Keywords: Urethra; Urethral stricture; Urologic surgical procedures

This is an Open Access article distributed under the terms of the Creative Commons Attribution Non-Commercial License (http://creativecommons.org/licenses/by-nc/4.0) which permits unrestricted non-commercial use, distribution, and reproduction in any medium, provided the original work is properly cited.

\section{INTRODUCTION}

Pelvic fracture urethral injuries (PFUI) in men are challenging clinical problem for urologists. In particular, pelvic bone fractures accompanying urethral injuries after traumatic accident are not easily accessible due to the

Received: 16 September, 2019 - Accepted: 5 October, 2019

Corresponding Author: Jong Jin Oh (iD https://orcid.org/0000-0003-0448-5992

Department of Urology, Seoul National University Bundang Hospital, Seoul National University College of Medicine, 82 Gumi-ro 173beon-gil, Bundang-gu, Seongnam 13620, Korea

TEL: +82-31-787-7351, FAX: +82-31-787-4057, E-mail: bebsuzzang@naver.com 
distorted anatomy [1,2]. Furthermore, many studies show a high recurrence rate for urethral stenosis after bulbomembranous anastomotic urethroplasty, which makes the treatment of posterior urethral injuries a reconstructive dilemma for urologic surgeons [2-4]. Even when proper preparation for bulbomembranous anastomotic urethroplasty, such as thorough preoperative evaluation, appropriate surgical planning, and adherence to basic surgical principles, is carried out, many studies still show unfavorable outcomes after bulbomembranous anastomotic urethroplasty in men with urethral injuries that are associated with pelvic bone injury [5-7]. In general, the recurrent rate of posterior urethral stenosis after bulbomembranous anastomotic urethroplasty is $14 \%$ to $25 \%$ [4,7,8]. More recently, Breyer et al. [9] reported that the overall primary stenosis-free survival rates at 1 , 3 , and 5 years were $88 \%, 82 \%$, and $79 \%$, respectively. In the case of posterior urethral injuries with pelvic bone fractures, the recurrence rate is $13.1 \%$ in Italy and $21.4 \%$ in India [6]; however, this study is not a pure representation of the recurrence rate bulbomembranous anastomotic urethroplasty because the data included other surgical methods such as urethrotomy and urethrostomy. Thus, the recurrence rate of urethral stenosis associated with pelvic bone injury after bulbomembranous anastomotic urethroplasty has not been well established.

As previous studies have emphasized, the outcomes after bulbomembranous anastomotic urethroplasty are influenced not only by defect length but also by several other factors, such as the cause of injuries [9,10]. In addition, PFUI was produced most commonly as a consequence of pelvic bone fracture, which may occur in up to $25 \%$ of cases [11,12]. If not managed properly, this can lead to a lifelong condition with deleterious consequences that may compromise not only the ability to urinate and maintain urinary continence but also fertility $[13,14]$. Until now, the surgical outcomes of urethral injuries in the setting of pelvic bone fractures are not well known. This study focuses on and analyzes the impact of pelvic bone fractures in urethral injury by evaluating and comparing the outcomes and differences in stenosis recurrence rate after bulbomembranous anastomotic urethroplasty in two groups divided based on the presence of pelvic bone fractures.

\section{MATERIALS AND METHODS}

\section{Patients}

This study was approved retrospectively by the Institutional Review Board of CHA Medical Center (approval number: CHAIRB013). The requirement for written consent was waived because of the retrospective study. We retrospectively analyzed the medical charts of patients evaluated and treated for posterior urethral injuries at a single institution by a single surgeon from December 2001 to May 2017. All patients underwent simultaneous retrograde urethrography (RUG) and combined cystourethrography (CCUG), and only men with posterior urethral injury were included in this study. These patients had received bulbomembranous anastomotic urethroplasty for posterior urethral injury. In total, 197 male patients with posterior urethral injury were included in the study. We excluded patients with less than a 1-year follow-up period or an anterior urethral stenosis. We also excluded patients who had an atonic bladder on the preoperative urodynamic study or who had missing relevant data. The 197 eligible patients were stratified into two groups according to the co-existence of pelvic bone fracture. There were 92 patients who had posterior urethral stenosis with pelvic bone fracture, and the other 105 patients had no accompanying pelvic bone fracture.

\section{Surgical technique}

All bulbomembranous anastomotic urethroplasty were performed by a single surgeon. All patients were placed in a lithotomy position and made an inverted Y-shaped perineal incision. The distal urethral end was identified by retrograde passage of a metallic urethral sound and the proximal urethral end was identified by antegrade passage of a metallic urethral sound through the suprapubic cystostomy tract. After measuring the extent of the urethral stenosis, all fibrotic tissues of the urethral stenosis including any peri-urethral scar tissue were excised completely. To avoid tension on the suture site, urethral anastomosis was performed by using a progressive perineal approach for mid-line separation of the proximal corporal bodies, inferior pubectomy, and supracorporal urethral rerouting. If scar tissue extended above the supra-montanal prostate, combined abdominal perineal exposure was created through a midline infra-umbilical incision. The proximal and distal ends of the urethra were spatulated and an anastomosis between the two ends was performed over a 16-Fr silicon urethral catheter using $4-0$ or $5-0$ Vicryl sutures. We sometimes used gracilis muscle flap (GMF) to reduce dead space after posterior urethral anastomosis. The skin incision was made parallel to the long axis of the gracilis muscle of the left thigh. The gracilis muscle was dissected from the medial aspect of the left thigh and released from its insertion. The GMF was rotated, and its distal end was brought to the perineal anastomotic urethra, and the perineal defect was filled with the rotated GMF. In most cases, suprapubic cystostomy was performed simultaneously. At 
3 weeks after the bulbomembranous anastomotic urethroplasty, pericatheter RUG was performed. If there was no leakage of contrast media, the urethral Foley catheter was removed. Otherwise, the Foley catheter was left in place, and the pericatheter RUG was repeated 1 to 2 weeks later. The suprapubic tube was clamped and removed only when the patient showed a reasonably good ability to void through the urethra.

\section{Outcomes analysis}

The patient variables that were collected preoperatively included age, cause of injuries, previous operation history and body mass index (BMI). The operation duration, the amount of blood loss estimated by calculating the blood amount collected in the suction container as well as the difference in the weights of dry and blood soaked sponges, and any perioperative complications described in the medical records were thoroughly reviewed. Urethral defect length (cm), which was defined as the injured longitudinal length to repair, was measured using a CCUG. Recurrence was de- fined as the need for any postoperative intervention such as urethrotomy or repeat urethroplasty. Additional postoperative interventions, which were mainly endoscopic internal urethrotomies, were conducted in the patients complaining of voiding discomfort and who had Qmax less than $15 \mathrm{~mL}$ per second, greater than $150 \mathrm{~mL}$ post-voiding residual urine, or a narrowed urethral caliber on follow-up RUG. We also defined postoperative incontinence as the need for pads and intermittent leakage of urine during abdominal straining. In addition, impotence was defined as being unable to perform sexual intercourse. We investigated outcomes after bulbomembranous anastomotic urethroplasty and the recurrence of urethral stenosis according to the presence of pelvic bone fractures. Secondly, we investigated the functional outcomes of incontinence and impotence after bulbomembranous anastomotic urethroplasty according to the presence of pelvic bone fracture. Postoperative surgical complications were defined according to the Clavien classification system [14].

The data are presented as means \pm standard deviation. Student t-test or the Mann-Whitney U-test was used for con-

Table 1. Demographic characteristics of study patients

\begin{tabular}{|c|c|c|c|}
\hline Characteristic & No pelvic bone fracture $(n=105)$ & Pelvic bone fracture $(n=92)$ & $\mathrm{p}$-value \\
\hline Age $(y)$ & $42.61 \pm 15.42$ & $39.25 \pm 14.76$ & 0.956 \\
\hline Body mass index $\left(\mathrm{kg} / \mathrm{m}^{2}\right)$ & $25.59 \pm 3.20$ & $22.14 \pm 3.53$ & 0.311 \\
\hline Defect length $(\mathrm{cm})$ & $2.68 \pm 1.65$ & $2.69 \pm 1.53$ & 0.956 \\
\hline Baseline creatinine (ng/dL) & $0.98 \pm 0.22$ & $0.99 \pm 0.65$ & 0.931 \\
\hline Cause of urethral injury & & & $<0.001$ \\
\hline Car accident & $18(17.1)$ & $50(54.3)$ & \\
\hline Crushing accident & $3(2.9)$ & $37(40.2)$ & \\
\hline Straddle & $60(57.1)$ & $5(5.4)$ & \\
\hline latrogenic & $24(22.9)$ & $0(0.0)$ & \\
\hline Diabetes mellitus & $2(1.9)$ & $1(1.1)$ & 0.550 \\
\hline Hypertension & $1(1.0)$ & $1(1.1)$ & 0.717 \\
\hline Site of urethral stenosis & & & 0.147 \\
\hline Including penile urethra & $31(29.5)$ & $14(15.2)$ & \\
\hline Including bulbous urethra & $105(100.0)$ & $92(100.0)$ & \\
\hline Including membranous urethra & $72(68.6)$ & $92(100.0)$ & \\
\hline Including prostatic urethra & $4(3.8)$ & $16(17.4)$ & \\
\hline Timing of urethroplasty & & & $<0.001$ \\
\hline Immediate & $16(15.2)$ & $0(0.0)$ & \\
\hline Delay & $89(84.8)$ & $92(100.0)$ & \\
\hline Previous urethral manipulation & $44(41.9)$ & $33(35.9)$ & 0.246 \\
\hline \multicolumn{4}{|l|}{ Urethral lengthening procedures } \\
\hline Urethral mobilization & $105(100.0)$ & $92(100.0)$ & 0.533 \\
\hline Corporeal separation & $46(43.8)$ & $45(48.9)$ & 0.279 \\
\hline Inferior pubectomy & $30(28.6)$ & $38(41.3)$ & 0.046 \\
\hline Rerouting of the urethra & $19(18.1)$ & $27(29.3)$ & 0.048 \\
\hline Gracilis muscle flap & $17(16.2)$ & $8(8.7)$ & 0.078 \\
\hline
\end{tabular}

Values are presented as mean \pm standard deviation or number (\%). 
tinuous data to evaluate comparisons between the groups. The chi-squared test was used for categorical data. Recurrencefree duration of urethral stenosis was evaluated using the Kaplan-Meier method. Cox proportional hazards regression analysis was performed to identify multivariable predictors of recurrence rate of urethral stenosis. Hazard ratios (HRs) were calculated to provide a relative risk between variables. All pvalues were set at 0.05 to define statistical significance. Statistical analyses were performed using IBM SPSS Statistics for Windows version 19.0 (IBM Corp. Armonk, NY, USA).

\section{RESULTS}

Among the 197 patients, 92 had pelvic bone fracture and 105 did not have pelvic bone fracture. The mean age was $41.0 \pm 15.1$ years, the mean urethral defect size was $2.6 \pm 1.5 \mathrm{~cm}$, and 77 patients (39.1\%) had a history of previous urethral surgeries at another hospital.

As shown in Table 1, urethral defect length was not significantly different between the two groups (2.68 vs. $2.69 \mathrm{~cm}$, $\mathrm{p}=0.956)$. Most patients in the pelvic bone fracture group had mechanical traumatic events, including car accidents and crushing accidents, but most of the patients in non-pelvic bone fracture group had straddle trauma accidents $(p<0.001)$. The patients with pelvic bone fracture were more likely to have involvement of the prostatic urethra compared to the intact pelvic bone group (3.8\% vs. $17.4 \%$ ). There was no significant difference in previous urethral manipulation between the pelvic bone fracture group (33/92, 35.9\%) and the intact pelvic bone group (44/105, 41.9\%). Furthermore, when considering methods of urethral lengthening, the patients with pelvic bone fracture underwent inferior pubectomy and rerouting of the urethra more of ten $(28.6 \%$ vs. $41.3 \%$, $\mathrm{p}=0.046 ; 18.1 \%$ vs. $29.3 \%$, $\mathrm{p}=0.048$ ).

Intra-operative and post-operative outcomes are summarized in Table 2. Estimated blood loss and operation duration were not significantly different between the two groups. Although the mean Qmax was not significantly different between the two groups, $(23.14 \mathrm{~mL} / \mathrm{s}$ vs. $24.76 \mathrm{~mL} / \mathrm{s}, \mathrm{p}=0.364)$ the urethral stenosis recurrence rate was higher in the pelvic bone fracture group (42/92, 45.7\%) than in the intact pelvic bone group (27/105, 25.7\%). The incidence rate of grade $\mathrm{II}-\mathrm{IV}$ complications after bulbomembranous anastomotic urethroplasty was $1.9 \%$ in the intact pelvic bone group and $5.4 \%$ in the pelvic bone fracture group, depending on the definition of Clavien classification $(\mathrm{p}=0.154)$.

There was no significant difference in the ability to maintain urinary continence as measured 3 months after bulbomembranous anastomotic urethroplasty between the two patient groups ( $5.7 \%$ vs. $12.0 \%$, $\mathrm{p}=0.096)$, but a significantly higher rate of impotence was shown in the pelvic bone fracture group compared to the intact pelvic bone group ( $22.9 \%$ vs. $66.3 \%, \mathrm{p}<0.001)$.

The recurrence rate of urethral stenosis according to

Table 2. Perioperative outcomes according to presence of pelvic bone fracture

\begin{tabular}{|c|c|c|c|}
\hline Characteristic & No pelvic bone fracture $(n=105)$ & Pelvic bone fracture $(n=92)$ & p-value \\
\hline \multicolumn{4}{|l|}{ Intra-operative complication } \\
\hline Estimated blood loss (mL) & $623.5 \pm 383.2$ & $719.7 \pm 1,061.7$ & 0.718 \\
\hline Operation time (min) & $152.6 \pm 65.5$ & $164.7 \pm 33.7$ & 0.076 \\
\hline Duration of catheterization (d) & $22.6 \pm 4.2$ & $23.0 \pm 4.8$ & 0.523 \\
\hline \multicolumn{4}{|l|}{ At 3 months post-operative } \\
\hline Mean Qmax (mL/s) & $23.14 \pm 10.96$ & $24.76 \pm 12.54$ & 0.364 \\
\hline Mean voiding volume (mL) & $202.82 \pm 134.51$ & $245.70 \pm 178.80$ & 0.058 \\
\hline Post-operation extravasation & $19(18.1)$ & $23(25.0)$ & 0.171 \\
\hline Recurrence of urethral stricture & $27(25.7)$ & $42(45.7)$ & 0.003 \\
\hline Clavien classification & & & 0.154 \\
\hline $\mathrm{I}-\mathrm{II}$ & 56 & 49 & \\
\hline III-IV & 2 & 5 & \\
\hline Re-operation procedure & & & 0.159 \\
\hline Visual urethrotomy & $27(25.7)$ & $25(27.2)$ & \\
\hline Redo-urethroplasty & $3(2.9)$ & $0(0.0)$ & \\
\hline Suprapubic cystostomy & $2(1.9)$ & $0(0.0)$ & \\
\hline \multicolumn{4}{|l|}{ At 3 months post-operative } \\
\hline Incontinence & $6 / 105(5.7)$ & $11 / 92(12.0)$ & 0.096 \\
\hline Impotence & $24 / 105$ (22.9) & $61 / 92(66.3)$ & $<0.001$ \\
\hline
\end{tabular}

Values are presented as mean \pm standard deviation, number (\%), or number only. 
the presence of a pelvic bone fracture was compared by Kaplan-Meier analysis (Fig. 1). During a median follow-up duration of 23.6 months, the recurrence rate at 5 years was significantly lower in the pelvic bone fracture group than in the intact pelvic bone group ( $40.9 \%$ vs. $27.4 \%$, $\mathrm{p}=0.003$ ).

To investigate the predictors for recurrence of urethral stenosis after bulbomembranous anastomotic urethroplasty, a Cox proportional hazard analysis was conducted and showed that the presence of pelvic bone injury was a significant predictor for the outcome of bulbomembranous anastomotic urethroplasty after adjusting for several factors, such as age, BMI, urethral defect length, urethral defect site, and previous urethral manipulation. Table 3 shows to identify predictive factor for recurrence of urethral stenosis. Pelvic bone injury is a significant risk factor for recurrence of urethral stenosis (HR, 2.155; 95\% confidence interval [CI], 1.145-4.057; $\mathrm{p}=0.017$ ). Furthermore, urethral defect length (HR, 1.339; 95\% CI, 1.097-1.636; $\mathrm{p}=0.004)$ and previous urethral manipulation (HR, 2.159; 95\% CI, 1.184-3.938; $\mathrm{p}=0.012$ ) were also significant risk factors for recurrence of urethral stenosis after bulbomembranous anastomotic urethroplasty.

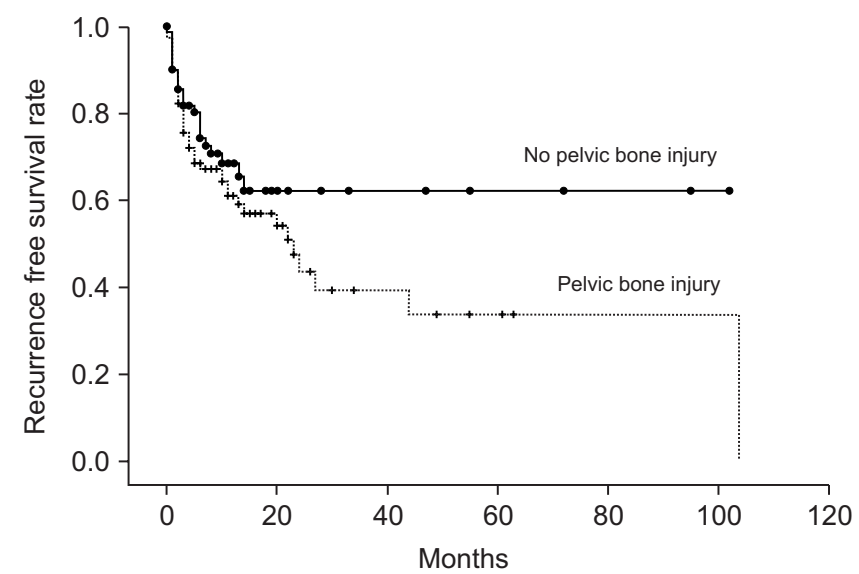

Fig. 1. Recurrence free rate of urethral stenosis following bulbomembranous anastomotic urethroplasty's in men who had posterior urethral injury with pelvic bone fracture and without.

\section{DISCUSSION}

Although there have been many studies investigating the etiology of posterior urethral injuries, few studies have focused on the results of bulbomembranous anastomotic urethroplasty according to the presence of pelvic bone injury $[3,15,16]$. In this study, bulbomembranous anastomotic urethroplasty in men with pelvic bone injuries had fewer successful outcomes than in men without pelvic bone injuries. Even after adjusting for factors that influenced the recurrence rate, such as age, urethral defect length, defect site and history of previous urethral manipulation, the presence of pelvic bone fractures was still a significant factor that predicted the recurrence of urethral stenosis in patients who underwent bulbomembranous anastomotic urethroplasty.

Bulbomembranous anastomotic urethroplasty still remains the gold standard therapeutic method for posterior urethral injuries because of its low rates of stricture recurrence [17]. In a systematic review, the overall posterior urethral stenosis recurrence rate after bulbomembranous anastomotic urethroplasty was $17.5 \%$ between 2000 and 2008 [18]. In meta-analysis results, surgical treatment of bulbomembranous anastomotic urethroplasty was accompanied by a success rate of $82 \%$ to $95 \%$ [19]. In some nations, bulbomembranous anastomotic urethroplasty is the most cost-effective therapeutic modality for repeat endourological intervention [20]. In this study, the overall recurrence rate after bulbomembranous anastomotic urethroplasty was approximately $35.0 \%$, but in cases with pelvic bone fracture, the urethral stenosis recurrence rate was $45.7 \%$, which was higher than the rate for all patients in this study. This higher urethral stenosis recurrence rate was likely due to the fact that many patients had already received previous urethroplasties or endoscopic urethral surgeries at other institutions (77/197, 39.1\%). In addition, the strict definition used for recurrence of urethral stenosis in our study might have resulted in a higher rate of recurrence.

Factors that can predict the success of bulbomembranous anastomotic urethroplasty are not still well established. Roehrborn and McConnell [21] attempted to identify factors

Table 3. Cox proportional hazard model predicting recurrence of urethral stenosis after bulbomembranous anastomotic urethroplasty

\begin{tabular}{lccc}
\multicolumn{1}{c}{ Variable } & Hazard ratio & 95\% Cl & $\mathrm{p}$-value \\
\hline Age & 1.018 & $0.997-1.039$ & 0.093 \\
Body mass index $\left(\mathrm{kg} / \mathrm{m}^{2}\right)$ & 0.998 & $0.415-2.400$ & 0.996 \\
Urethral defect length $(\mathrm{cm})$ & 1.339 & $1.097-1.636$ & 0.004 \\
Previous urethral manipulation (yes or no) & 2.159 & $1.184-3.938$ & 0.012 \\
Pelvic bone injury (yes or no) & 2.155 & $1.145-4.057$ & 0.017 \\
\hline
\end{tabular}

$\mathrm{Cl}$, confidence interval. 
that may predict failure of urethroplasty, and they found that the factor with the greatest impact on urethroplasty outcome was previous manipulation of the urethra. Similarly, Breyer et al. [9] argued that prior urethroplasty (HR, 1.8; 95\% CI, 1.1-3.1; $\mathrm{p}=0.03$ ) and failed endoscopic therapy (HR, 1.7 ; $95 \%$ CI, 1.0-3.0; $p=0.04$ ) were predictive of treatment failure. Scarberry et al. [2] argued that previous intervention in the form of endoscopic realignment or urethroplasty had adverse effects on the success rate of anastomotic urethroplasty $(\mathrm{p}<0.05)$. They also argued that stenosis length and patient age did not influence the outcome of urethroplasty. In our study, previous urethral manipulation was also a significant predictor for recurrence of urethral stenosis. These results indicate that healthy tissue adjacent to the urethra is an important factor for maintaining urethral patency. However, the aforementioned study did not evaluate pelvic bone injury, so, to our knowledge, our study is the first study to investigate the effect of pelvic bone injury in men undergoing of bulbomembranous anastomotic urethroplasty.

Surgical technique is also an important factor in the success of urethroplasty. Koraitim [22] asserted that incomplete excision of scar tissue, inadequate fixation of the prostatic mucosa, and anastomotic tension were predictive of surgical failure. In our study, urethral defect length (HR, 1339; 95\% CI, 1.097-1.636; $\mathrm{p}=0.004$ ), prostatic urethra injury (HR, 1.041; 95\% CI, 1.01-1.06), and pelvic bone injury (HR, 2.155; 95\% CI, 1.145-4.057; $\mathrm{p}=0.017$ ) were predictive of urethral stenosis recurrence. Unlike other studies, we showed that urethral defect length and the presence of pelvic bone fracture are important factors and significant predictors for urethral stenosis recurrence.

Pelvic bone fractures causing disruption at the bulbomembranous junction remain the main cause of posterior urethral injury. The optimal timing of surgery (emergency or delayed) and methods of surgical intervention (open or endoscopic) are still unclear. The debate revolves around the risk of late complications, which may occur as a direct consequence of injury or because of initial treatment. Moreover, the impact of pelvic bone fractures on bulbomembranous anastomotic urethroplasty is not clear. To that regard, this study has shown that pelvic bone fractures are closely related to recurrence of urethral stricture.

Vascularity and the degree of injury are the most important factors for success of urethroplasty [23]. Generally, the normal urethra and spongiosum tissue must have sufficient innervation and microvasculature to maintain their elastic and compliant properties [24]. However, an injured urethra loses its relationship between the smooth muscle, extracellular matrix, and vascular sinusoid [25]. After a urethral in- jury with a pelvic bone fracture, the urethra and surrounding pelvic tissues are injured by trauma, which leads to a decrease in vascularity; thus, the periurethral tissues are no longer supplied with vascularity. The initial injury recovers by forming a surrounding hematoma and fibrosis, and these factors make accurate identification of the anatomical planes difficult, requiring meticulous dissection. As a result, impaired vascularity and persistent inflammation prevent continuous healing of the urethra [26]. Furthermore, a study on microvascular damage caused by smoking in men who underwent urethroplasty also revealed environmental factors affecting the surrounding urethra tissue, which were important factors affecting success [9].

For post-operative complications, the patients with pelvic bone fracture had a higher rate of impotence than those without pelvic bone fracture at 3 months postoperatively $(22.9 \%$ vs. $66.3 \%, \mathrm{p}<0.001)$. Although our study differed in its time at evaluation, the impotence rate was similar to other papers [27-29], which reported that the incidence of impotence ranged from $60 \%$ to $72 \%$ when assessment was performed 3 to 15 months after pelvic bone injury. Still, Koraitim [30] argued that spontaneous recovery of sexual function could occur up to 2 years after injury as a result of neuropraxis and the rehabilitation of accessory penile arterial supply. So far, several risk factors for impotence after pelvic bone injury have been identified. Malavaud et al. [14] presented significant potential factors, including pubic diastasis. Anger et al. [27] suggested that posterior urethral disruption and/or other injuries associated with urethral disruption have a significant impact on cavernous nerves.

Our study has several limitations. First, its retrospective nature may have caused selection bias. Although we adjusted for several factors that influenced the outcomes, there were still unconfirmed factors that might have influenced the outcomes of the study. This could be addressed by conducting a prospective, large-scale, cohort study. Secondly, this study has as its limitation the presence of significant numbers of cases with straddle or iatrogenic injury in the posterior urethral stenosis group without pelvic bone fracture. This group consisted mostly of patients with stenosis extending from the distal membranous urethra to the bladder neck secondary to repeated endourologic procedures and previous bulbomembranous anastomotic urethroplasty's as the primary etiology, although these are less likely to cause posterior urethral stenosis. This limitation is due to an extremely lack of experienced physicians in Korea who can perform appropriate procedures once posterior urethral stenosis occurs and the position of this medical center as a referral hospital. Another limitation is the difference between 
the baseline characteristics of the two groups, including etiology, time of bulbomembranous anastomotic urethroplasty, and the type of procedures used for urethral lengthening. Since these differences may affect functional outcomes, the corresponding differences make assessment of the results difficult and may deteriorate the credibility of the results. Lastly, among the complications, we could not use validated questionnaires to assess erectile function and continence preoperatively and postoperatively. The recording of urinary and sexual function was not based on qualitative or semiquantitative measurement. It also should be validated by well-designed prospective studies.

\section{CONCLUSIONS}

The patients with posterior urethral injuries associated with pelvic bone fracture had a higher rate of urethral stenosis recurrence after perineal bulbomembranous anastomotic urethroplasty than patients without pelvic bone fracture. We postulated that the higher recurrence rate of urethral injury in the group with pelvic bone fractures was associated with micro-environmental factors, which included the inflammatory response and microvascular vulnerability. These results should be validated by a prospective, largescale, cohort study.

\section{CONFLICTS OF INTEREST}

The authors have nothing to disclose.

\section{AUTHORS' CONTRIBUTIONS}

Research conception and design: Don Kyoung Choi and Jong Jin Oh. Data acquisition: Don Kyoung Choi, Jong Jin Oh, and Sungj̈n Kim. Statistical analysis: Don Kyoung Choi and Jong Jin Oh. Data analysis and interpretation: Don Kyoung Choi, Jong Jin Oh, and Sungjin Kim. Drafting of the manuscript: Don Kyoung Choi and Jong Jin Oh. Critical revision of the manuscript: Don Kyoung Choi and Jong Jin Oh. Administrative, technical, or material support: Don Kyoung Choi, Jong Jin Oh and Sungjin Kim. Supervision: Jong Jin Oh. Approval of the final manuscript: all authors.

\section{REFERENCES}

1. Min BD, Lee ET, Kim WT, Kim YJ, Yun SJ, Lee SC, et al. Tubal buccal mucosa graft without anastomosis of the proximal urethra for long segment posterior urethral defect repair. Korean J Urol 2012;53:737-40.
2. Scarberry K, Bonomo J, Gómez RG. Delayed posterior urethroplasty following pelvic fracture urethral injury: do we have to wait 3 months? Urology 2018;116:193-7.

3. Horiguchi A, Shinchi M, Masunaga A, Okubo K, Kawamura K, Ojima K, et al. Primary realignment for pelvic fracture urethral injury is Associated with Prolonged Time to Urethroplasty and Increased Stenosis Complexity. Urology 2017;108:184-9.

4. Zou Q, Zhou S, Zhang K, Yang R, Fu Q. The immediate management of pelvic fracture urethral injury-endoscopic realignment or cystostomy? J Urol 2017;198:869-74.

5. Mathur R, Aggarwal G, Satsangi B, Khan F, Odiya S. Prognosis of urethral strictures following pelvic fracture urethral distraction defects--a single centre study. Int J Surg 2011;9:68-71.

6. Kulkarni SB, Barbagli G, Kulkarni JS, Romano G, Lazzeri M. Posterior urethral stricture after pelvic fracture urethral distraction defects in developing and developed countries, and choice of surgical technique. J Urol 2010;183:1049-54.

7. Kessler TM, Schreiter F, Kralidis G, Heitz M, Olianas R, Fisch M. Long-term results of surgery for urethral stricture: a statistical analysis. J Urol 2003;170:840-4.

8. Berger AP, Deibl M, Bartsch G, Steiner H, Varkarakis J, Gozzi C. A comparison of one-stage procedures for post-traumatic urethral stricture repair. BJU Int 2005;95:1299-302.

9. Breyer BN, McAninch JW, Whitson JM, Eisenberg ML, Mehdizadeh JF, Myers JB, Voelzke BB. Multivariate analysis of risk factors for long-term urethroplasty outcome. J Urol 2010;183:613-7.

10. Andrich DE, Day AC, Mundy AR. Proposed mechanisms of lower urinary tract injury in fractures of the pelvic ring. BJU Int 2007;100:567-73.

11. Gómez RG, Mundy T, Dubey D, El-Kassaby AW, Firdaoessaleh, Kodama R, et al. SIU/ICUD consultation on urethral strictures: pelvic fracture urethral injuries. Urology 2014;83(3 Suppl):S48-58.

12. Koraitim MM, Marzouk ME, Atta MA, Orabi SS. Risk factors and mechanism of urethral injury in pelvic fractures. Br J Urol 1996;77:876-80.

13. Koraitim MM, Atta MA, Fattah GA, Ismail HR. Mechanism of continence after repair of post-traumatic posterior urethral strictures. Urology 2003;61:287-90.

14. Malavaud B, Mouzin M, Tricoire JL, Gamé X, Rischmann P, Sarramon JP, et al. Evaluation of male sexual function after pelvic trauma by the International Index of Erectile Function. Urology 2000;55:842-6.

15. Flynn BJ, Delvecchio FC, Webster GD. Perineal repair of pelvic fracture urethral distraction defects: experience in 120 patients during the last 10 years. J Urol 2003;170:1877-80.

16. Andrich DE, Mundy AR. The nature of urethral injury in cases of pelvic fracture urethral trauma. J Urol 2001;165:1492-5. 
17. Andrich DE, Mundy AR. What is the best technique for urethroplasty? Eur Urol 2008;54:1031-41.

18. Meeks JJ, Erickson BA, Granieri MA, Gonzalez CM. Stricture recurrence after urethroplasty: a systematic review. J Urol 2009;182:1266-70.

19. Hosseini J, Tavakkoli Tabassi K. Surgical repair of posterior urethral defects: review of literature and presentation of experiences. Urol J 2008;5:215-22.

20. Rourke KF, Jordan GH. Primary urethral reconstruction: the cost minimized approach to the bulbous urethral stricture. J Urol 2005;173:1206-10.

21. Roehrborn CG, McConnell JD. Analysis of factors contributing to success or failure of 1-stage urethroplasty for urethral stricture disease. J Urol 1994;151:869-74.

22. Koraitim MM. Failed posterior urethroplasty: lessons learned. Urology 2003;62:719-22.

23. Mundy AR. Transperineal bulbo-prostatic anastomotic urethroplasty. World J Urol 1998;16:164-70.

24. Cavalcanti AG, Yucel S, Deng DY, McAninch JW, Baskin LS. The distribution of neuronal and inducible nitric oxide synthase in urethral stricture formation. J Urol 2004;171:1943-7.
25. Cavalcanti AG, Costa WS, Baskin LS, McAninch JA, Sampaio FJ. A morphometric analysis of bulbar urethral strictures. BJU Int 2007;100:397-402.

26. Wadhwa SN, Chahal R, Hemal AK, Gupta NP, Dogra PN, Seth A. Management of obliterative posttraumatic posterior urethral strictures after failed initial urethroplasty. J Urol 1998;159:1898-902.

27. Anger JT, Sherman ND, Dielubanza E, Webster GD, Hegarty PK. Erectile function after posterior urethroplasty for pelvic fracture-urethral distraction defect injuries. BJU Int 2009;104:1126-9.

28. Shenfeld OZ, Kiselgorf D, Gofrit ON, Verstandig AG, Landau $\mathrm{EH}$, Pode D, et al. The incidence and causes of erectile dysfunction after pelvic fractures associated with posterior urethral disruption. J Urol 2003;169:2173-6.

29. Harwood PJ, Grotz M, Eardley I, Giannoudis PV. Erectile dysfunction after fracture of the pelvis. J Bone Joint Surg Br 2005;87:281-90.

30. Koraitim MM. Predictors of erectile dysfunction post pelvic fracture urethral injuries: a multivariate analysis. Urology 2013;81:1081-5. 